\title{
Suppression of Thymidine Uptake of Human Lymphocytes by Co-trimoxazole
}

\author{
P. M. GAYLARDE， I. SARKANY
}

British Medical fournal, 1972, 3, 144-146

\section{Summary}

Co-trimoxazole (trimethoprim-sulphamethoxazole) causes a decrease in the uptake of labelled thymidine in lymphocytes cultured in the presence of phytohaemagglutinin. This phenomenon was observed in $60 \%$ of 25 subjects. In cultures affected by the drug the mean suppression was $84 \%$. A small decrease in thymidine uptake was noted with trimethoprim and sulphamethoxazole separately, but the effect was much more pronounced with the combination of the two drugs. The mechanism responsible for this phenomenon is discussed. The action is probably not due to the ability of these drugs to interfere with folic acid metabolism and it is likely that there is no direct effect on DNA synthesis.

The suppression of thymidine uptake by lymphocytes in vitro in the presence of co-trimoxazole may not have any obvious clinical significance. However, in view of a report of an immunosuppressive action of trimethoprim in mice, it is possible that the leucopenia observed in some patients treated with this drug may have been caused by a similar mechanism.

These experiments show that lymphocytes in vitro are suppressed by co-trimoxazole in concentrations comparable to, or smaller than, those found in vivo under normal therapeutic conditions. They are therefore likely to be clinically relevant.

\section{Introduction}

In vitro, in the presence of phytohaemagglutinin, human peripheral blood lymphocytes undergo transformation into large blast cells which are capable of division and incorporate labelled thymidine into their nucleic DNA. Transformation may also be produced by antigens to which the donor's lymphocytes are sensitized (tuberculin, smallpox vaccine, grass pollen, etc.). Lymphoblast transformation and increased thymidine uptake occur in a certain group of drug hypersensitivity reactions (Holland and Mauer, 1964; Sarkany, 1967). There is always a small amount of thymidine incorporation in the presence of drugs even in the absence of associated lymphocyte transformation. Little attention has been paid to the phenomenon of the uptake of thymidine in vitro in negative reactions.

While studying lymphocyte transformation in response to drugs in cases of hypersensitivity reactions, we observed that on three occasions in the presence of co-trimoxazole there was a decrease in the uptake of labelled thymidine compared with control cultures. Suppression of DNA synthesis in vitro is rare in the presence of drugs in concentrations normally found in the patient's blood and used for in-vitro testing. As a result of this observation we set up an investigation of this phenomenon.

\footnotetext{
Department of Dermatology, the Royal Free Hospital, London W.C.1

P. M. GAYLARDE, B.sC., Research Assistant

I. SARKANY, F.R.C.P., Consultant
}

\section{Methods}

Heparinized blood was taken from 25 normal subjects and allowed to stand at $37^{\circ} \mathrm{C}$ for about one hour. The supernatant plasma was withdrawn and diluted with 4 volumes of Eagle's basal medium. Then $2-\mathrm{ml}$ aliquots were put in tubes and $0.1 \mathrm{ml}$ of phytohaemagglutinin was added to each. The effect of the drugs sulphamethoxazole and trimethoprim was studied individually and in combination at different concentrations added at the beginning of the experiment. In addition, in seven cultures the effect of adding the drugs for the last four hours of incubation was investigated. The effect of adding folic acid in addition to the drugs was assessed. Most of the experiments were carried out using duplicate or triplicate cultures.

After 68 hours in culture $1 \mu \mathrm{Ci}$ of tritiated thymidine was added and the cells were harvested four hours later. The cells were washed with trichloroacetic acid and saline and collected by filtration on Millipore filters. After drying they were placed in a xylene/butyl PBD scintillator and counted by means of a Packard scintillation counter. In three cultures ${ }^{32} \mathrm{P}$-labelled phosphate was added and the incorporation into DNA of the cells was measured.

Similar cultures were also set up containing small numbers of red blood cells, and smears were made after 72 hours for morphological studies. The effect of two other drugs, using similar culture conditions, was examined. On two separate occasions plasma was pooled from four individuals and cultures containing penicillin in concentrations from 3,200 to $200 \mathrm{IU} / \mathrm{ml}$ in twofold dilutions and sulphadiazine 400 to $50 \mu \mathrm{g} / \mathrm{ml}$ in twofold dilutions were set up and compared in triplicate with control cultures. The effect of suppression of dihydrofolate reductase by $0.25 \mu \mathrm{g}$ of methotrexate $/ \mathrm{ml}$ was investigated in three cultures.

\section{Results}

Lymphocytes from 25 subjects were cultured in the presence of trimethoprim $1 \mu \mathrm{g} / \mathrm{ml}$ and sulphamethoxazole $5 \mu \mathrm{g} / \mathrm{ml}$. Decreased ${ }^{3} \mathrm{H}$-thymidine uptake was noted in 15 of these, and the distribution of the results is shown in Fig. 1. Average suppression for these 15 cultures was $84 \%$. The suppression of ${ }^{32} \mathrm{P}-$ labelled phosphate caused by co-trimoxazole was similar to that of labelled thymidine.

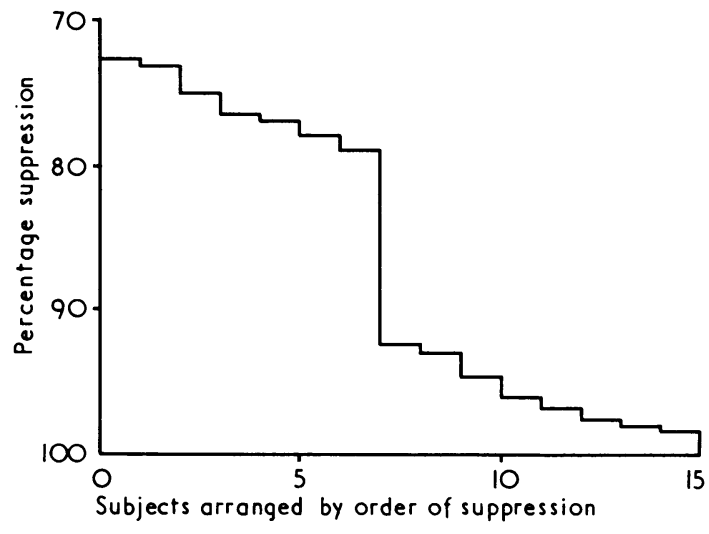

FIG. 1-Distribution of percentage suppression of ${ }^{3} \mathrm{H}$ thymidine uptake produced by trimethoprim $1 \mu \mathrm{g} / \mathrm{ml}$ and sulphamethoxazole $5 \mu \mathrm{g} / \mathrm{ml}$. 
The effect of concentrations between 1.25 and $20 \mu \mathrm{g}$ of sulphamethoxazole $/ \mathrm{ml}$ and 0.25 and $4 \mu \mathrm{g}$ of trimethoprim $/ \mathrm{ml}$ in twofold dilutions, in a ratio of 5 parts sulphamethoxazole to 1 part trimethoprim, was investigated in six cases. Of these, four showed suppression, and the average curve of these is shown in Fig. 2. Extrapolation of the values on the curve shows that the suppression does not reach $100 \%$ as the drug concentration increases towards infinity.

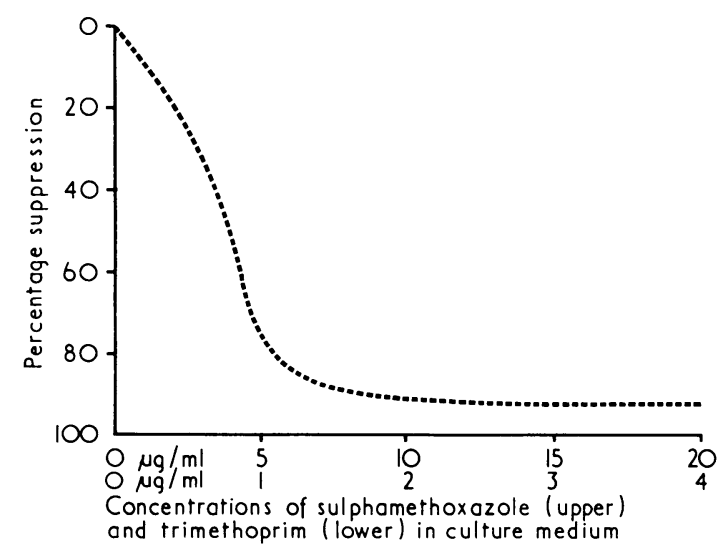

FIG. 2-Degree of suppression of ${ }^{3} \mathrm{H}$ thymidine uptake caused by different concentrations of trimethoprim and sulphamethoxazole.

Cultures of lymphocytes in the presence of various concentrations of trimethoprim and sulphamethoxazole separately showed that a small but definite decrease in thymidine uptake occurred in the presence of each of the drugs. Together, at the same concentrations, a very large decrease occurred.

The number of cells remaining after three days was similar in the cultures treated with co-trimoxazole and in controls. There was no obvious microscopical change in the morphology of the cells and no changes suggestive of toxicity were noted. The degree of transformation was not affected by the drugs and the only obvious alteration was a decrease in the number of mitoses.

The addition of folic acid at a level of $40 \mu \mathrm{g} / \mathrm{ml}$ produced no reversal of the suppression caused by co-trimoxazole. The addition of co-trimoxazole at the same time as the addition of thymidine caused no inhibition of uptake even in those cultures where suppression occurred when co-trimoxazole was added at the beginning of the culture.

There was no significant alteration in the uptake of ${ }^{3} \mathrm{H}$ thymidine by lymphocytes cultured in the presence of high concentrations of penicillin, sulphadiazine, or methotrexate.

\section{Discussion}

These results show that co-trimoxazole produced a suppression of the uptake of ${ }^{3} \mathrm{H}$-thymidine in 15 out of 25 normal subjects. In the 15 individuals the suppression of thymidine uptake was between 73 and $98.5 \%$, with a mean value of $84 \%$. This represents a very severe degree of suppression, since only onesixth of the normal amount of labelled thymidine is taken up by the cells in the presence of the drug. No significant change took place in the remaining subjects. Of four other patients previously tested for suspected drug hypersensitivity to cotrimoxazole, suppression of thymidine uptake had been found in three. This observation prompted our investigation of the 25 normal subjects reported here.

The explanation for this phenomenon is not clear. Interference with folic acid metabolism seems a possible cause for the diminished thymidine uptake. Studies on human folate reductase have shown that for liver-derived enzyme $50 \%$ inactivation is caused by a $3 \times 10^{-4} \mathrm{M}$ concentration of trimethoprim (Bushby and Hitchings, 1968). Normal blood levels are approximately at the $1 \times 10^{-5} \mathrm{M}$ level and a $50 \%$ reduction in dihydrofolate reductase activity in sensitive bacteria is brought about at a concentration of about $10^{-8} \mathrm{M}$. The concentration of trimethoprim used in our studies is only $1 / 100$ of that which produced $50 \%$ inhibition of human tetrahydrofolate reductase. This drug cannot produce a significant effect on folate metabolism, unless the enzyme in lymphocytes is readily inhibited or the lymphocytes are able to concentrate trimethoprim within their cytoplasm. Further evidence against the hypothesis of folate reductase inhibition is the fact that suppression of thymidine uptake is not reversed by the addition of large amounts of folic acid.

The inhibition of folate reductase interferes with DNA synthesis by stopping the synthesis of DNA precursors, most significantly that of thymine. The addition of thymidine to a culture lacking in this precursor would result in a very high initial rate of thymidine incorporation into DNA. A small but statistically insignificant increase in thymidine uptake occurred with the addition of methotrexate to lymphocyte cultures.

It was thought that the drug combination might interfere with the uptake of the labelled thymidine into the lymphocytes by interference with the active transport of the labelled substance into the cell. Phosphate incorporation, however, is affected in the same fashion as thymidine incorporation and therefore this explanation for the suppression of uptake may be ruled out. The addition of co-trimoxazole towards the end of the culture period showed that more than four hours needed to elapse before these drugs could exert an effect on DNA synthesis. This makes it unlikely that the suppression is mediated by a direct effect on DNA or the enzymes responsible for its synthesis.

Trimethoprim and sulphamethoxazole individually are known to have a bacteriostatic effect on bacterial metabolism. The sulphonamide component interferes with folic acid synthesis by competing with $p$-aminobenzoic acid whereas trimethoprim is a competitive inhibitor of dihydrofolic acid reductase. Trimethoprim thus prevents the synthesis of thymine, and the bacteriostatic action is antagonized by the presence of thymidine. However, in combination the two drugs have a bactericidal effect, but the mechanism of this is not understood. One can speculate that this bactericidal action is based on a mechanism similar to the suppression of mitosis demonstrated in our work.

The fact that suppression of thymidine in transformed lymphocytes was not found in all subjects is also intriguing. The distribution pattern shown in Fig. 1 suggests that even among the group showing suppression of thymidine uptake the results can be divided into two subgroups. Of the 15 subjects whose cells showed suppression, the results in seven were between 73 and $79 \%$ and in the remainder between 92.5 and $98.5 \%$. The suggestion that the results cluster around certain values, thus forming two groups, gives rise to speculation that there is an intrinsic factor in the cells which is responsible and that perhaps this factor is genetically determined.

Hulme and Reeves (1971) drew attention to leucopenia associated with co-trimoxazole after renal transplantation and showed that this could be profound and sustained. All the affected patients were receiving azathioprine. Haematological changes during therapy with co-trimoxazole in typhoid fever in children were reported by Scragg and Rubidge (1971), who therefore recommended monitoring of the blood picture when the drug was used. These findings may be relevant in view of our demonstration of the ability of this drug combination to interfere with DNA metabolism. It is conceivable that such interference may affect not only lymphocytes but the white cell series as a whole, and consequently it is possible that the finding of leucopenia in various clinical studies is due to this effect. Although we have found interference only with DNA meta- 
bolism in vitro, others (Ghilchik et al., 1970) have shown that trimethoprim has an immunosuppressive effect in vivo in mice.

In some earlier trials with combinations of trimethoprim and sulphonamides other than sulphamethoxazole, minor haematological changes were noted (Bushby, 1970), but these were considered not to represent a real therapeutic hazard. Tests showed that the changes were not due to interference with folic acid metabolism and they were ascribed to the sulphonamides. Our results also fail to demonstrate abnormalities of folic acid metabolism but they show that sulphamethoxazole can interfere with cell division whereas sulphadiazine does not do this even at concentrations 400 times greater.
We thank Miss B. Bradley for technical help. We also thank the Herbert E. Dunhill Trust for support.

\section{References}

Bushby, S. R. M. (1970). South African Medical fournal, Suppl. No. 3. Bushby, S. R. M., and Hitchings, G. H. (1968). British fournal of Pharmacology and Chemotherapy, 33, 72 .

Ghilchik, M. W., Morris, A. S., and Reeves, D. S. (1970). Nature, 277, 393.

Holland, P., and Mauer, A. M. (1964). Lancet, 1, 1368.

Hulme, B., and Reeves, D. S. (1971). British Medical fournal, 3, 610.

Sarkany, I. (1967). Lancet, 1, 743.

Scragg, J. N., and Rubidge, C. J. (1971). British Medical fournal, 2, 738

\title{
Induction of Abortion by Extra-amniotic Administration of Prostaglandins $\mathbf{E}_{2}$ and $\mathbf{F}_{2} \alpha$
}

\author{
M. P. EMBREY, KEITH HILLIER, P. MAHENDRAN
}

British Medical fournal, 1972, 3, 146-149

\section{Summary}

The use of prostaglandins $E_{2}$ and $F_{2} \alpha$, administered by extra-amniotic instillation, for the induction of abortion was studied in 94 patients in the first and second trimesters of pregnancy. Abortion was successfully induced in $87 \%$ of patients within 36 hours and in $94 \%$ within 48 hours. The mean abortion time was 22.4 hours. In $60 \%$ of patients abortion was complete.

Though the differences were not statistically significant, on average multigravid patients aborted more quickly than primigravidae, while the mean abortion time in PGE $_{2}$-treated patients was less than in those receiving $\mathrm{PGF}_{2} \alpha_{\text {. }}$

No serious complications occurred. Some side effects were observed. Occasional vomiting was the commonest symptom but the incidence of side effects was lower than with alternative routes of administration. A leucocytosis was often noted but there were no significant instances of infection.

The method has proved a safe and effective means of terminating pregnancies in the second trimester.

\section{Introduction}

There is now considerable evidence concerning the significant oxytocic properties of the prostaglandins. Independent studies have shown the potential value of prostaglandins of the $E$ and $F \alpha$ series $\left(\mathrm{PGE}_{2}\right.$ and $\left.\mathrm{PGF}_{2} \alpha\right)$ both as parturigenic agents (Embrey, 1969, 1970a; Beazley et al., 1970; Karim et al., 1970; Roth-Brandel and Adams, 1970) and as abortifacients (Embrey 1970b; Karim and Filshie, 1970a, 1970b).

These studies were restricted initially to the intravenous route of administration but the prolonged intravenous infusions necessary for induction of abortion commonly caused severe side effects-notably diarrhoea and vomiting (especially with

\footnotetext{
Nuffield Department of Obstetrics and Gynaecology, the Radcliffe Infirmary, University of Oxford, Oxford

M. P. EMBREY, F.R.C.S., F.R.C.o.G., First Assistant

KEITH HILLIER, B.SC., PH.D., Nuffield Research Officer

P. MAHENDRAN, M.B., D.OBST.R.C.O.G., Senior House Officer (On study leave from the Malaysian Armed Forces)
}

$\mathrm{PGF}_{2} \alpha$ ) and venous erythema (especially with $\mathrm{PGE}_{2}$ ). Attempts to shorten the infusion time by using larger doses were disappointing and resulted only in an intolerable level of side effects (Hillier and Embrey, 1972).

Other routes of administration have, therefore, been explored, and we (Embrey and Hillier, 1971) described an intrauterine (extra-amniotic) technique. The present paper reports an extended trial of the method.

\section{Patients and Methods}

One hundred and twenty patients referred for therapeutic abortion were studied. Twenty-six had received some additional form of therapy - for example, intra-amniotic glucose, saline, or intravenous oxytocin-and though their response to treatment and side effects were no different, they have been excluded, leaving for analysis 94 patients who received prostaglandin alone until abortion occurred or up to 36 hours. After 36 hours the case was considered a treatment failure and intravenous oxytocin administered.

Without anaesthesia a Foley catheter, 14-16 French gauge, was introduced through the cervix with the aid of a speculum (and, if necessary, a single-tooth vulsellum), so that the inflatable balloon lay just within the internal os. The balloon volume varied with gestational age from about $20 \mathrm{ml}$ at 8 weeks to 30 $\mathrm{ml}$ at 12 weeks and $40 \mathrm{ml}$ at 16 weeks and over.

The extra-amniotic catheter and a small-bore "manometer" connecting tube were filled with saline containing ampicillin $(1 \mathrm{mg} / \mathrm{ml})$ and connected to a three-way tap. A syringe inserted into the three-way tap was used for making the prostaglandin injections, and the same tap, if required, was connected to a pressure transducer and Devices two-channel recorder to register intrauterine pressure changes.

Prostaglandin Administration.-Prostaglandin $\mathrm{E}_{2}\left(\mathrm{PGE}_{2}\right)$ or prostaglandin $\mathrm{F}_{2} \alpha\left(\mathrm{PGF}_{2} \alpha\right)$ was used. $\mathrm{PGF}_{2} \alpha$ was supplied in a sterile trometamol solution and was diluted to give a $250-\mu \mathrm{g} / \mathrm{ml}$ solution in sterile water or isotonic saline. $\mathrm{PGE}_{2}$ supplied as a powder was dissolved in 0.5 to $1 \mathrm{ml}$ of absolute alcohol and made up to $100 \mu \mathrm{g} / \mathrm{ml}$ with distilled water or isotonic saline. The latter was then sterilized by cold ultrafiltration and an aliquot taken for culture. On proof of sterility the solution was used within 48 hours. An initial test dose of $50 \mu \mathrm{g}$ of $\mathrm{PGE}_{2}$ or $250 \mu \mathrm{g}$ of $\mathrm{PGF}_{2} \alpha$ was given into the extra-amniotic space, taking into account the dead-space volume of the Foley catheter and connector (approximately $4.5 \mathrm{ml}$ ). If no adverse effects occurred a 